Reviews 


\title{
Paradoxical Role of HMGB1 in Pancreatic Cancer: Tumor Suppressor or Tumor Promoter?
}

\author{
MARÍA JOSÉ GARCÍA CEBRIÁN ${ }^{1}$, MONIKA BAUDEN ${ }^{1}$, ROLAND ANDERSSON ${ }^{1}$, \\ STEFAN HOLDENRIEDER ${ }^{2}$ and DANIEL ANSARI ${ }^{1}$ \\ ${ }^{1}$ Department of Surgery, Clinical Sciences Lund, Lund University and Skåne University Hospital, Lund, Sweden; \\ ${ }^{2}$ Institute of Laboratory Medicine, German Heart Center at the Technical University Munich, Munich, Germany
}

\begin{abstract}
Pancreatic cancer has a dismal prognosis and there is an increasing and unmet need to identify better diagnostic and therapeutic targets in order to ameliorate the course of the disease. HMGB1, a nuclear DNA-binding protein that acts as a transcription factor, is currently in the limelight. HMGB1 exhibits a dual role in pancreatic cancer; when intracellular, it acts as an anti-tumor protein stabilizing the genome, whereas extracellular HMGB1 behaves as a protumor protein with cytokine, chemokine and growth factor functions. Although the exact mechanisms of HMGBI in pancreatic cancer are still to be elucidated, the significance of this protein for processes, such as autophagy, immunogenic cell death, tumor growth, metastasis and resistance to chemotherapy, have become increasingly clear. In this review, we provide a systematic summary and review of the biological and clinical relevance of HMGBI in pancreatic cancer.
\end{abstract}

Pancreatic adenocarcinoma represents the fourth leading cause of death from cancer in the Western world with a 5-year relative survival rate of less than $7 \%$ (1). The disease is projected to become the second leading cause of cancer death by the year 2020, after lung cancer, if no progress is made in its management (2). The reasons for this negative trend include lack of methods for early diagnosis and the absence of effective treatment. The development of biomarkers for pancreatic

This article is freely accessible online.

Correspondence to: Daniel Ansari, MD, Ph.D., Department of Surgery, Clinical Sciences Lund, Lund University and Skåne University Hospital, Lund, SE-221 85 Lund, Sweden. Tel: +46 4622246 72, e-mail: daniel.ansari@med.lu.se

Key Words: HMGB1, pancreatic cancer, tumor suppressor, tumor promoter, biomarker, review. cancer is a rapidly expanding area within pancreatic cancer research. Biomarkers may allow early non-invasive detection of the disease. Furthermore, biomarkers may be used to predict prognosis and, potentially, also for targeted interventions.

The high mobility group box 1 (HMGB1) protein is a nuclear non-histone protein that can be released from the nucleus as a damage-associated molecular pattern (DAMP). Through a signaling pathway involving the receptor for advanced glycation end products (RAGE), HMGB1 has been associated with tumor progression due to its elevated expression in certain cancer types, especially pancreatic cancer (3). Likewise, HMGB 1 has been correlated to invasion and metastasis (4), resistance to chemotherapy agents $(5,6)$ and autophagy and immunogenic cell death (7).

The aim of this review was to bring together the described actions of HMGB1 in pancreatic cancer in order to achieve a deeper understanding of its role in the disease process and potential implications for improving diagnosis, prognosis and treatment.

\section{Methods}

A computerized search of the PubMed database of the National Library of Medicine from inception to 2015 was performed to identify all relevant articles published using the search terms 'HMGB1 pancreatic cancer'. The initial search was expanded using the 'related articles' function in PubMed. Titles and abstracts of all identified articles were studied and a selection was made based on their relevance to the subject. Reference lists within relevant articles were searched to identify publications not captured by computerized searches. Human studies were included. Experimental studies from animal and cell studies were also included when exploring mechanistic explanations for the role of HMGB1 in pancreatic cancer. A total of 54 articles regarding HMGB1 were included in the final analysis. 


\section{HMGB1: A Double-edged Sword}

HMGB1 is a nuclear DNA-binding protein that belongs to the High Mobility Group-box superfamily with a dual function: transcription and organization of DNA and extracellular signaling, being one of the main extracellular DAMP molecules. HMGB1 binds DNA through its two domains, A and $\mathrm{B}$ boxes. Aspartate and glutamate form the end-string with an acidic carboxyl terminus (8).

HMBG1 is essentially expressed in the nucleus of all eukaryotic cells and continuously transferred between the nucleus and cytoplasm. Acetylation and phosphorylation induce translocation of HMGB1 to the cytoplasm and increase the secretion from macrophages and cancer cells. Post-translational methylation of HMGB1 causes its cytoplasmic localization in neutrophils. Overexpression, as well as cytoplasmic localization of HMGB1, has been observed in most tumor cells. Moreover, HMGB1 plays a critical role in DNA repair and is related to a number of transcription factors implicated in cancer development, stimulating their activity. For instance, HMGB1 interacts directly with P53 (tumor suppressor) to enhance its DNA binding $(3,9)$.

HMGB1 can also function as a negative signaling molecule, mediating the response to infection and injury, thereby promoting inflammation. To act as a "danger signal" and inflammatory mediator, HMGB1 must be transported extracellularly. This occurs in two fundamentally different manners: active secretion from living inflammatory cells or passive release from necrotic or stressed cells. Once in the extracellular space, it binds to different receptors, such as RAGE and toll-like receptors (TLR)-2, TLR-4, TLR-9 (3).

Nevertheless, even if the mechanisms are not very well described yet, oxidative stress appears as a central regulator of HMGB1's translocation, release and activity in inflammation and cell death (10). Calcium- or reactive oxygen species (ROS)-dependent mechanisms were also confirmed to induce HMGB1 release (3).

Cells undergoing necrosis or tumor cells treated with chemotherapy release HMGB1, which possesses proinflammatory properties. HMGB 1 , released from dying tumor cells, stimulates mature dendritic cells (mDC) tumor antigen processing, favoring anticancer immune responses. Furthermore, activation of HMGB 1 and its receptor RAGE results in the activation of nuclear transcription factor NFkappa B (NF-kB), which up-regulates leukocyte adhesion molecules and the production of proinflammatory cytokines and angiogenic factors (3).

In many cases, extracellular HMGB1 acts as a pro-tumor protein due to its cytokine, chemokine and growth factor activity. It has been found that certain aminoacids in the B box domain are essential for the protein's proinflammatory cytokine function, especially the cysteine aminoacid in the position $106(\mathrm{C} 106)$ in the $\mathrm{B}$ box domain that is required for HMGB1 to bind to TLR-4 activating macrophages (11).

Dendritic cell activation by HMGB1 involves the reduced form of C106. The inflammatory capacity of HMGB1 is prevented when cysteines are oxidized to sulfonic acids, suggesting that the function of HMGB1 can be modulated through cysteine redox modifications (12). Fully reduced HMGB1 has no post-translational modifications and it is the predominant form in nucleus and cytoplasm in basal conditions and interacting with several receptors, such as CXCR4, to promote cell migration. HMGB1 is oxidized in the cytoplasm after ROS production (13). The oxidation of HMGB1 acts as a physiological negative feedback mechanism. Because the extracellular environment is mainly oxidative during inflammatory processes, the oxidation restricts the proinflammatory activity of HMGB1 preventing excessive inflammatory damage (12). After oxidation, sulfonyl HMGB1 has no activity for cell migration or cytokine induction (13).

Moreover, C23 and C45, two additional cysteine residues in HMGB1, form an intramolecular disulfide bond that stabilize the protein and may be implicated in HMGB1's inflammatory signaling pathway (12). Disulfide HMGB1 interacts with TLR4 to induce cytokine and chemokine transcription and secretion (13). The role of C23 and C45 is so crucial that, when fully reduced HMGB1 (with no posttranslational modifications) is moderately oxidized, even if the cytokine function would be inhibited, the present disulfide bond is still able - and needed - to induce its cytokine capacity. Additionally, when mutated, C23 and C45 avoid the initiation of autophagy by HMGB1 inhibiting the relation to Beclin1 (12, 13).

On the other hand, intracellular HMGB1 acts as antitumor protein due to its ability to sustain genome stability and autophagy activity during tumor growth (3). Importantly, it has been described that when HMGB1 is overexpressed, oncoproteins regulating cell proliferation increase, whereas tumor suppressor proteins decrease (14).

With regard to pancreatic cancer, the HMGB1/RAGE pathway has a crucial function in the regulation of apoptosis. Targeted knockdown of $R A G E$ in pancreatic tumor cells, leads to increased apoptosis, diminished autophagy and decreased tumor cell survival. Furthermore, depletion of HMGB1 in pancreatic tumor cell renders them significantly more sensitive to induced apoptotic cell death (3).

Moreover, RAGE and HMGB1 jointly enhance tumor cell mitochondrial complex I activity and, therefore, ATP production, needed for supporting anabolism and tumor cell proliferation and migration (4).

Increasing evidence, thus, suggests that cellular localizations of RAGE and HMGB1 affect their function and interaction. RAGE and HMGB1 appear in their soluble form in the normal tissues, whereas they are insoluble and membrane-bound in 
cancer cells where they form a stable complex (15). Of note, soluble forms of these proteins are detectable in the bloodstream of cancer patients, representing potential diagnostic biomarkers, as well as therapeutic targets (16).

\section{HMGB1, Autophagy and Apoptosis in Pancreatic Cancer}

Autophagy is an ubiquitous process in mammalian cells that results in the breakdown of cytoplasmic components within the lysosome in response to stress conditions, such as starvation, allowing the cell to adapt to environmental or developmental changes (17). Autophagy is generally recognized as programmed cell survival mechanism maintaining miscellaneous physiological processes. On the other hand, however, autophagy is also implicated in the pathogenesis of several diseases, including cancer (18).

The role of autophagy in pancreatic cancer is complex as it is associated with both tumor suppression and increased tumorigenesis $(19,20)$. Pancreatic cancer cells exhibit constitutive autophagy under basal conditions. When autophagy is inhibited in pancreatic cancer cells, ROS increase, causing DNA damage and a reduction of mitochondrial oxidative phosphorylation. This results in significant growth suppression of pancreatic cancer cells in vitro leading to tumor regression (21).

Moreover, ROS trigger the translocation and release of HMGB1 in pancreatic cancer cells. Disulfide HMGB1 binds to RAGE, induces Beclin 1-dependent autophagy (the intramolecular disulfide bridge in HMGB1 is needed to bind to Beclin1 and sustain autophagy) and promotes tumor cell resistance to chemotherapeutic agents or ionizing radiation. In contrast, oxidized HMGB1 increases the cytotoxicity of these agents and induces apoptosis via the mitochondrial pathway $(22,23)$.

In a hypoxic microenvironment, hypoxia-induced autophagy mediates survival of pancreatic tumor-initiating cells. Physiological enhanced levels of autophagy render pancreatic cancer stem-like cells resistant to induction of apoptosis by hypoxia and starvation, while inhibition of autophagy induces death of pancreatic cancer stem-like cells and inhibits self-renewal potential (18).

The HMGB1-RAGE pathway plays critical roles in the induction of autophagy and inhibition of HMGB1-RAGE leads to increased apoptosis and decreased autophagy in pancreatic cancer cells. RAGE inhibits apoptosis through P53 in response to chemotherapy. P53 has a dual function. Nuclear P53 stimulates autophagy in a transcription-dependent manner, whereas cytoplasmic P53 inhibits the formation of autophagosomes in a transcription-independent fashion (24, 25). Furthermore, studies have shown that p53-deficient mice form markedly fewer autophagosomes (26). Moreover, RAGE sustains autophagy by relating to certain molecules involved in the formation of the autophagosome as it decreases phosphorylation of the mammalian target of rapamycin (mTOR) or increases Beclin 1-Vps34 interaction $(24,27)$. These results can be extrapolated to HMGB1, as it has been found that endogenous HMGB1 is a critical pro-autophagic protein that enhances cell survival and limits programmed apoptotic cell death (22).

Murine pancreas containing an activated oncogenic allele of Kras, the most common mutational event in pancreatic cancer, develops a small number of pre-cancerous lesions that stochastically advance into pancreatic cancer over time. In mice containing oncogenic Kras and lacking p53, loss of autophagy no longer blocks tumor progression, but actually accelerates tumor onset (26).

RAGE is required for extracellular HMGB1-mediated autophagy. Antioxidant enzymes (e.g., superoxide dismutases) and small-molecule antioxidants (e.g., N-acetyl-L-cysteine) inhibit HMGB1 activation, as well as autophagy in pancreatic cancer cells. In the nucleus, HMGB1 regulates expression of heat shock protein beta-1 (HSPB1). Recent data suggest that HSPB1 inhibits apoptosis and its phosphorylation promotes autophagy (25).

When analyzing the relationship between autophagy and pancreatic cancer risk factors, only one connection has been outlined so far. It has been found that pancreatitis stimulates autophagy induction and, at the same time, impairs late stages of autophagy (18). A defective autophagy could cause vacuole accumulation and trypsinogen activation, contributing to the development of pancreatitis (28).

Seaweed polyphenols suppress the transcription of all investigated autophagy regulators, including HMGB1, in human and mice pancreatic cancer cell lines in vitro and in vivo. Moreover, the data collected strongly suggest that the selected polyphenols could serve as effective adjuvants for current pancreatic cancer treatment modalities and may inhibit tumor relapse (29).

\section{HMGB1 and Immunogenic Cell Death}

Immunogenic cell death (ICD) is a form of cell death that stimulates an anti-tumor immune response against dead-cell antigens, derived from cancer cells (30). Multiple stimuli can trigger ICD, which involves changes in the composition of the cell surface, as well as release of soluble mediators, occurring in a defined temporal sequence. In fact, ICD is preceded or accompanied by the emission of a series of immunostimulatory DAMPs in a precise spatiotemporal configuration. ICDassociated biomarkers include, amongst others, HMGB1, the endoplasmic reticulum (ER) chaperone calreticulin (CALR) and ATP. Cell death that is not accompanied by CALR exposure, ATP secretion and HMGB1 release is generally not perceived as immunogenic, resulting in a partial or even total loss of immune system stimulation (31). 
Such signals operate on a series of receptors expressed by dendritic cells to stimulate the presentation of tumor antigens to $\mathrm{T}$ cells (32).

The notion that cancer cell apoptosis is detected by the immune system involves the possibility of a self anticancer response and also implies a critical role for immunostimulation in therapies against malignant cells (31).

As it has been previously mentioned, HMGB1 is considered to be an important DAMP and its release to the extracellular space seems to be essential for the optimal presentation of tumor antigens to dendritic cells and efficient $T$ cell stimulation $(19,20)$.

Nevertheless, it should be taken into consideration that a high release of nucleosomes and HMGB1, as well as low release of soluble RAGE (sRAGE), have been found to be associated with poorer prognosis (33). Soluble RAGE has a different role than cellular RAGE, acting as a decoy receptor that binds to sHMGB1, inhibiting its functions. In late tumor stages, high levels of sHMGB1 are associated with poor prognosis due to chronic adaptation. In fact, this would be occurring due to the reason that chronic ICD could lead to adaptation and down-regulation of the immune response, while acute ICD - also induced by some chemotherapeutic agents leads to immune stimulation and better patient outcome (34).

Immunogenic cell death can be stimulated by retinoic acidinducible gene I (RIG-I)-like helicase (RLH), an ubiquitously expressed cytosolic helicase that recognizes viral RNA species and can be activated by synthetic RNA. When treated with RIG-I ligands, efficient tumor control is observed, as well as the activation of dendritic cells and CD8+ T cells as effector, connecting RLH to immunogenic cell death triggering adaptive immunity. Markers of ICD, HMGB1 included, are highly released when pancreatic cancer cells are treated with RLH ligands (20).

The pathway involves type I interferon (IFN) $(7,20)$ with a double action; it alerts the immune system to danger leading to activation of dendritic and effector cells, while it stops inhibition of immunosuppressive cells, such as regulatory T cells (20). Local treatment of potentially resectable pancreatic cancers with RLH ligands that would carry a highly immunogenic form of cell death before surgery may facilitate the induction of a T-cellmediated antitumor immune response with the potential to control outgrowth of residual tumor cells (7).

As it has been previously described, the cure of particularly aggressive malignancies requires induction of ICD, coupling oncolysis with immune responses via calreticulin, ATP and HMGB1 release from dying tumor cells.

Novel therapies employing oncolytic viruses have emerged as promising anticancer approaches.

One interesting strategy involves the oncolytic parvovirus $\mathrm{H}-1$ (H-1PV), which activated multiple interconnected death pathways when targeting human pancreatic cells and elevated extracellular HMGB1 in all infected cultures, whether non- dying, necrotic or apoptotic. HMGB1 release was not promoted by additional oncolysis upon co-treatment with gemcitabine. This chemotherapeutic alone failed to stimulate HMGB1 release despite strong cytotoxic effects. HMGB 1 release in pancreatic cancer cells appears to follow the alternative cytokine secretory pathway (using, for instance, molecular transporters). Secreted HMGB1, therefore, functions as an alarm and might represent a general mechanism by which viral infection is signaled to the immune system. The oncolytic virus H-1PV, which is not pathogenic in humans, could be then regarded as a multimodal anticancer treatment (35).

\section{HMGB1 and Pancreatic Cancer Growth and Metastasis}

The response of the host stroma to the invasive tumor is remarkable in pancreatic cancer and important to recognize in order to understand tumor growth. This dynamic process is known as a desmoplastic reaction and involves a complex interaction between the normal host epithelial cells, invading tumor cells, stromal fibroblasts, inflammatory cells, proliferating endothelial cells, an altered extracellular matrix and growth factors activating oncogenic signaling pathways by autocrine and paracrine mechanisms (36).

Several key molecules have been identified, such as collagen type I, fibronectin, laminin, matrix metalloproteinases (MMP) and tissue inhibitors of MMP, as well as growth factors, such as transforming growth factor-beta (TGF $\beta$ ), platelet-derived growth factor (PDGF), connective tissue growth factor (CTGF) and hepatocyte growth factor (HGF), and also chemokines and integrins (36).

The tumor necrosis factor (TNF) superfamily ligandreceptor - of which the fibroblast growth factor (FGF)inducible 14 (Fn14) is a part of, is as well involved in many cellular processes including proliferation, migration, differentiation, inflammation and angiogenesis.

The Fn14 receptor is expressed at relatively low levels in normal tissues but dramatically elevated in a wide variety of tumor types, especially in pancreatic cancer. Pancreatic cancer cells treated with a compound composed of anti-Fn14 monoclonal antibody (ITEM-4) conjugated to recombinant gelonin (rGel), a highly cytotoxic ribosome-inactivating $N$ glycosidase, showed the highest release of HMGB1 compared to different cancer cell lines (e.g., breast, lung and bladder) (37).

The dismal prognosis of pancreatic cancer is partly due to its high risk of local invasion and metastasis. Moreover, metastasis is the major cause of mortality and morbidity in patients with cancer. HMGB1 has a role in the transcription of many genes involved in different steps in the metastatic cascade and has been linked to different types of cancer in human and animal models (38). The HMGB1 protein was proposed to be directly involved in tumor cell metastasis 
through its ability to promote cell migration, modulate the adhesive properties of cells and modify components of the extracellular matrix (15).

HMGB 1 has been identified as a gene involved in the metastatic phenotype of pancreatic cancer in several studies. An up-regulation of this gene with a subsequent overexpression of HMGB 1 was shown in cell lines derived from the metastatic lesions in patients with pancreatic cancer (39). Therefore, HMGB1 has been closely associated with tumorigenesis in the pancreas and suggested as a diagnostic marker for pancreatic cancer (40).

The crucial role of HMGB1 for the invasive properties of pancreatic cancer was further proven when transfecting antisense-HMGB1 expression vector into a human pancreatic cancer cell line. The antisense-HMGB1 inhibited the expression of HMGB1, as well as that of metalloproteinases $M M P-2$ and $M M P-9$ mRNA expression, observing then a significant reduction in cell migration (41).

Moreover, strong expression of the HMGB1 receptor, RAGE, was detected in human pancreatic carcinoma cells with high metastatic ability. On the contrary, low levels of RAGE were observed in cells with low ability. Similarly, expression of MMP-9 showed nearly the same tendency. RAGE and MMP-9 are expressed concordant with the metastatic capacity of the human pancreatic cancer cells (42), although the connection between them has not yet been found.

RAGE, and its ability to regulate gene expression through NF-kB, constitutively activated in pancreatic cancer, was proposed as one of the possible mechanisms involved in pancreas cancer metastasis (43).

Most importantly, systemic in vivo administration of small RAGE antagonist peptide (RAP) blocking receptor activation by multiple ligands (S100P, S100A4 and HMGB-1) reduced the growth and metastasis of pancreatic tumors. Besides, RAP inhibited the interaction of S100P, S100A4 and HMGB-1 with RAGE at micromolar concentrations. Finally, RAP also reduced the ability of the ligands to stimulate RAGE activation of NF-kB in cancer cells in vitro and in vivo. (44).

Therefore, targeting RAGE may represent a promising tool for future treatment of pancreatic cancer.

\section{Gemcitabine Resistance}

The standard treatment for advanced pancreatic cancer is gemcitabine (Gem). However, the clinical effect of Gem is very limited due to an inherent and acquired chemoresistance, becoming, therefore, a hindrance to effective treatment. ICD is one of the keys to improve the efficacy of anticancer treatment; however, the capacity of Gem to induce ICD is currently debated (45).

Firstly, considering that hypoxia is one of the hallmarks of pancreatic cancer, the impact of hypoxia inducible factor-1 inhibitor, PX-478, has been investigated showing that, when combined with Gem, the anti-tumor effect is significantly enhanced as it stimulates ICD (45).

Secondly, studies on the relationship between miRNAs and the chemosensitivity of tumors have received increasing attention in recent years. miR-218 expression, one of the main miRNAs involved in cancer, is down-regulated in human pancreatic cancer tissues and cell lines. Additionally, miR-218 expression is significantly lower in Gem-resistant cells compared to the sensitive ones, thus indicating that the resistance of pancreatic cancer cells to Gem might be related to miR-218. It has also been demonstrated that overexpression of miR-218 enhances the sensitivity of pancreatic cancer cells to Gem (5). HMGB1 is also related to the sensitivity of pancreatic cancer cells to Gem-based chemotherapy (35) and miR-218 negatively regulates the expression of HMGB1, inhibiting the proliferation and invasion of pancreatic cancer cells (6). Consequently, the mechanism of the miR-218 effect may depend on the regulation of HMGB1 expression (5) and stimulating miR-218 might show positive results in pancreatic cancer patients (6).

Finally, up-regulated HMGB1 was found in Gem-resistant pancreatic cancer cells, supporting, once again, the association of high HMGB1 and poor prognosis. HMGB1, as a nuclear transcription factor, is similarly recognized to regulate the expression of heat-shock protein 27 (HSP27), a key player in gemcitabine-resistance, implicating HMGB1 as the activator of the HSP27 pathway in Gem-resistance $(46,47)$.

\section{HMGB1: A Potential Diagnostic and Prognostic Biomarker for Pancreatic Cancer?}

It has been found that increased quantities of HMGB1, as well as low serum levels of sRAGE, are associated with an unsatisfactory response to therapy and worse prognosis. HMGB1 could, thus, be regarded as a potential diagnostic and prognostic/predictive biomarker for pancreatic cancer (48), although cancer antigen (CA) 19-9 (the only established serum biomarker for pancreatic cancer to date) and cytokeratin-19 fragments (CYFRA 21-1) were proven remarkably predictive in a similar patient setting (49). Even if immunogenic biomarkers are not as powerful as these molecules, they should still be taken into consideration with regard to chemotherapy response evaluation and patient prognosis (48).

However, HMGB1 has its limitations. Serum HMGB1 can be elevated in other cancers or in pancreatic inflammatory diseases, such as acute pancreatitis (50), although, in these inflammatory conditions, levels of HMGB1 are distinctively lower. To solve the previous problem, HMGB1 can be measured alongside with CA 19-9, increasing the specificity and sensitivity $(33,51)$.

Tissue samples of pancreatic cancer patients after surgical tumor resection were analyzed to investigate the prognostic role of immunohistochemical HMGB1 and HMGB2 
expression. The study showed that nuclear HMGB1-positive and HMGB2-negative expression was a powerful and potentially independent negative indicator of disease-specific survival in operated patients and, by extension, a novel prognostic marker, especially within the first 2 years after surgery (52). Even though it is believed that HMGB2 has similar effects and roles as HMGB1 in cancer development, it functions reportedly as a potential tumor suppressor gene. It has consequently a key role in preventing malignant transformation by inducing cell-cycle arrest and/or apoptosis, especially in the setting of pancreatic cancer in a very similar way as P53, although it is not clearly described (52).

Regarding the HMGB1 receptor, RAGE levels measured in blood samples of pancreatic cancer patients undergoing systemic chemotherapy were found to be significantly higher in patients with stable or remissive disease (53). This fact correlates to the previous information as soluble RAGE acts differently from cellular RAGE preventing immunostimulatory effects rather than promoting them, i.e. functioning as a decoy receptor (34).

\section{Discussion}

In pancreatic cancer, HMGB1 exhibits a dual and paradoxical role. Extracellular HMGB1 becomes a pro-tumor protein, acting as cytokine, chemokine and growth factor, whereas intracellular HMGB1 functions as an anti-tumor protein, stabilizing the genome and sustaining autophagy (Figure 1). Moreover, patient stage should be taken into consideration when targeting HMGB1 as, for instance, extracellular HMGB1 may contribute to ICD-associated antitumor immunity in the early stages of the disease, but promote residual tumor cell survival in the late stages due to the adaptation and subsequent inhibition of the immune response when ICD becomes a chronic process (54).

With regard to HMGB1 intracellular functions, it binds to DNA and regulates several different processes, being as well one of the main proteins responsible for epigenetic changes (55). HMGB1 level are significantly raised in patients with pancreatic cancer, hence proving a valuable indicator of course of treatment, prognosis and, perhaps, even diagnosis. Comeasurement with the current tumor marker CA19-9 could be performed to further increase specificity and sensitivity for pancreatic cancer detection as HMGB1 is highly elevated, not only in pancreatic cancer, but also in other tumors and benign conditions (56).

In addition, suppression of intracellular HMGB 1 expression inhibits autophagy and increases apoptosis, which stimulates the efficiency of different therapies. Endogenous HMGB1 is a pro-autophagic protein, enhancing cell survival and limiting apoptotic cell death. It is released with sustained autophagy, late apoptosis and necrosis. In pancreatic cancer cells, autophagy is constitutively activated (22). When autophagy is inhibited in pancreatic cancer cells, tumor regression is observed, as there is a decrease of mitochondrial phosphorylation due to DNA damage caused by ROS activation. Likewise, ROS trigger the translocation and release of extracellular HMGB 1 in pancreatic cancer cells. Disulfide HMGB1, in its normal form, binds to RAGE and induces autophagy, promoting tumor resistance to therapeutic strategies. In contrast, HMGB1 oxidized by ROS increases the cytotoxicity of these agents and induces apoptosis via the mitochondrial pathway (23). Doubletreatment approach could, therefore, be possible to stimulate tumor regression: either by blocking HMGB1 release or favoring its oxidation.

Knowledge of the signal transduction and regulation of HMGB1 release is one of the hot topics in immunity and cancer research. HMGB1 can induce immune responses by itself. In addition, it might shift its activity from immune activation to immune tolerance (54). Immunogenic cell death constitutes one of the keys to determine the success of anticancer therapies as it stimulates an immune response against dead-cell antigens. HMGB1 is one of the main DAMPs released to the extracellular medium by dying cells. The activation of dendritic cells for $\mathrm{T}$ cell stimulation is one of the proposed mechanisms for immune activation, although the exact mechanism has not yet been described. Nevertheless, it is known that the response is stage-dependent: in earlier phases, an immune susceptible scenario is offered, while, in late stages of the disease, less susceptible conditions for immunogenic cell death are presented. Thus, combined therapeutic approaches are required.

At present, the standard anticancer therapy for pancreatic cancer is Gem. The capacity of Gem to induce ICD is currently debated. Potential combination therapies, such as HIF-1 inhibitor, PX-478, together with Gem targeting the hypoxic microenvironment within the tumor in order to stimulate ICD are being studied and described (45). Moreover, microRNAs are involved in the tumor sensitivity to Gem. miR-218 negatively regulates the expression of HMGB1, inhibiting the proliferation and invasion of pancreatic cancer cells. Further, this microRNA is down-regulated in pancreatic cancer $(5,6)$, also causing a higher resistance to Gem.

Apart from pancreatic cancer cell resistance to Gem, one of the main reasons for poor prognosis of disease is given by local invasion and metastasis of the tumor. $H M G B 1$ is responsible for the transcription of a wide range of proteins involved in the metastatic pathway. Furthermore, it favors metastasis by promoting cell migration and altering the extracellular matrix, thus showing a double implication, both intra and extracellular, in the process. HMGB1 and its receptor RAGE are up-regulated and overexpressed in cancer types with a high metastatic ability.

In conclusion, HMGB1 is unquestionably a crucial protein for the development and progression of pancreatic cancer. 


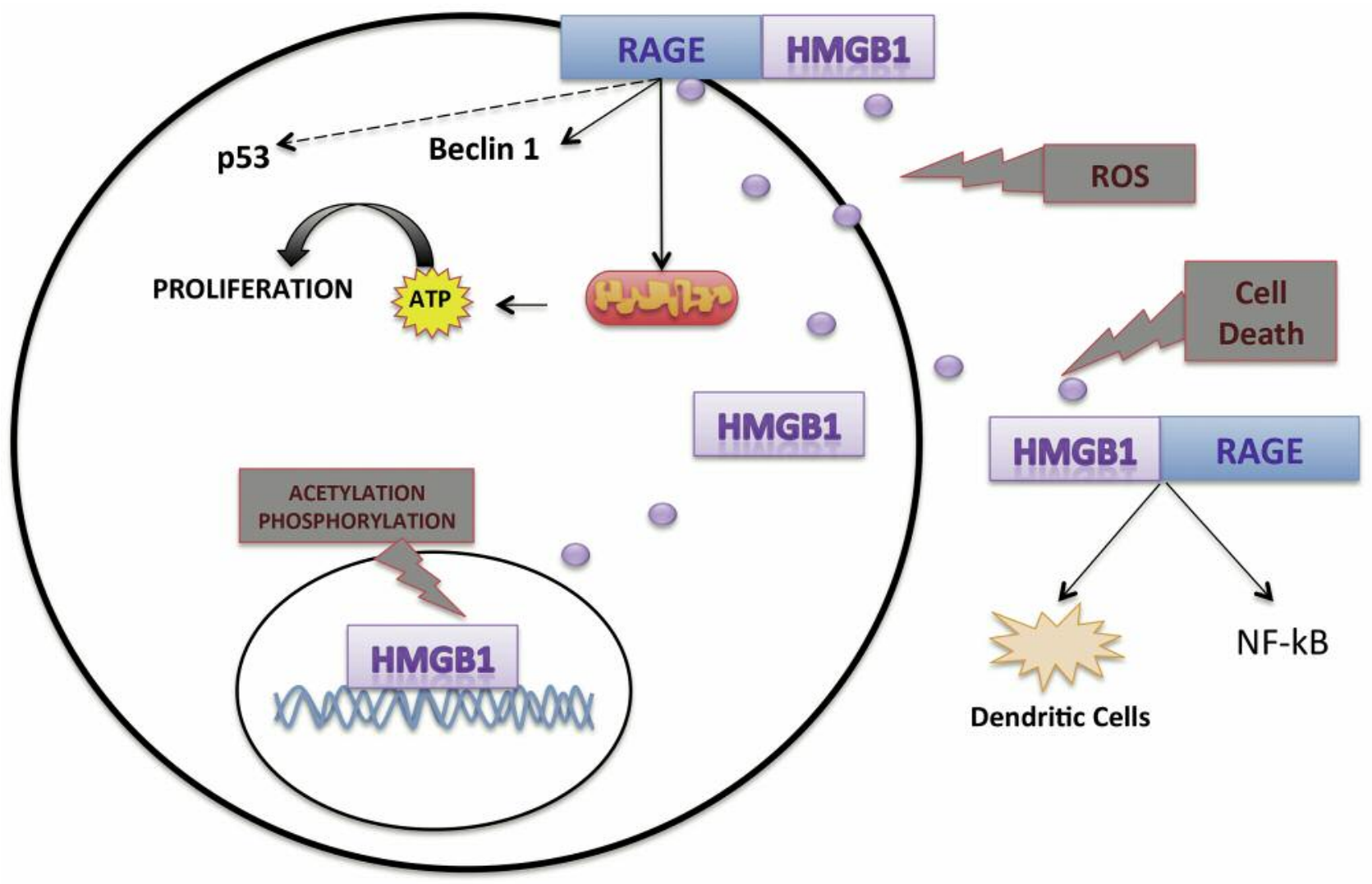

Figure 1. HMGB1 signaling pathways in pancreatic cancer.

While acting as a tumor suppressor in normal cells and earlystage pancreatic cancer, it may switch its role to facilitate tumor progression in advanced-stage pancreatic cancer. Understanding the exact mechanisms that trigger the tumorigenic effects of HMGB1 will be pivotal for the future development of treatments that target this protein.

\section{References}

1 Siegel RL, Miller KD and Jemal A: Cancer statistics, 2015. CA Cancer J Clin 65: 5-29, 2015.

2 Rahib L, Smith BD, Aizenberg R, Rosenzweig AB, Fleshman JM and Matrisian LM: Projecting cancer incidence and deaths to 2030: the unexpected burden of thyroid, liver, and pancreas cancers in the United States. Cancer Res 74: 2913-2921, 2014.

3 Tang D, Kang R, Zeh HJ, 3rd and Lotze MT: High-mobility group box 1 and cancer. Biochim Biophys Acta 1799: 131-140, 2010.

4 Kang R, Tang D, Schapiro NE, Loux T, Livesey KM, Billiar TR, Wang $H$, Van Houten $B$, Lotze MT and Zeh HJ: The HMGB1/RAGE inflammatory pathway promotes pancreatic tumor growth by regulating mitochondrial bioenergetics. Oncogene 33: 567-577, 2014.

5 Liu Z, Du R, Long J, Guo K, Ge C, Bi S and Xu Y: microRNA218 promotes gemcitabine sensitivity in human pancreatic cancer cells by regulating HMGB1 expression. Chin J Cancer Res 27: 267-278, 2015.

6 Liu Z, Xu Y, Long J, Guo K, Ge C and Du R: microRNA-218 suppresses the proliferation, invasion and promotes apoptosis of pancreatic cancer cells by targeting HMGB1. Chin J Cancer Res 27: 247-257, 2015.

7 Duewell P, Steger A, Lohr H, Bourhis H, Hoelz H, Kirchleitner SV, Stieg MR, Grassmann S, Kobold S, Siveke JT, Endres S and Schnurr M: RIG-I-like helicases induce immunogenic cell death of pancreatic cancer cells and sensitize tumors toward killing by CD8(+) T cells. Cell Death Differ 21: 1825-1837, 2014.

8 Bianchi ME, Beltrame M and Paonessa G: Specific recognition of cruciform DNA by nuclear protein HMG1. Science 243: 1056$1059,1989$.

9 Sims GP, Rowe DC, Rietdijk ST, Herbst R and Coyle AJ: HMGB1 and RAGE in inflammation and cancer. Annu Rev Immunol 28: 367-388, 2010.

$10 \mathrm{Yu}$ Y, Tang D and Kang R: Oxidative stress-mediated HMGB1 biology. Front Physiol 6: 93, 2015.

11 Yang H, Hreggvidsdottir HS, Palmblad K, Wang H, Ochani M, Li J, Lu B, Chavan S, Rosas-Ballina M, Al-Abed Y, Akira S, Bierhaus A, Erlandsson-Harris H, Andersson U and Tracey KJ: A critical cysteine is required for HMGB1 binding to Toll-like receptor 4 and activation of macrophage cytokine release. Proc Natl Acad Sci USA 107: 11942-11947, 2010. 
12 Yang H, Lundback P, Ottosson L, Erlandsson-Harris H, Venereau E, Bianchi ME, Al-Abed Y, Andersson U, Tracey KJ and Antoine DJ: Redox modification of cysteine residues regulates the cytokine activity of high mobility group box-1 (HMGB1). Mol Med 18: 250-259, 2012.

13 Antoine DJ, Harris HE, Andersson U, Tracey KJ and Bianchi ME: A systematic nomenclature for the redox states of high mobility group box (HMGB) proteins. Mol Med 20: 135-137, 2014.

14 Gong H, Zuliani P, Komuravelli A, Faeder JR and Clarke EM: Analysis and verification of the HMGB1 signaling pathway. BMC Bioinformatics 11(Suppl 7): S10, 2010.

15 Todorova $\mathrm{J}$ and Pasheva E: High mobility group B1 protein interacts with its receptor RAGE in tumor cells but not in normal tissues. Oncol Lett 3: 214-218, 2012.

16 Tesarova P, Cabinakova M, Mikulova V, Zima T and Kalousova M: RAGE and its ligands in cancer - culprits, biomarkers, or therapeutic targets? Neoplasma 62: 353-364, 2015.

17 Klionsky DJ: Autophagy: from phenomenology to molecular understanding in less than a decade. Nat Rev Mol Cell Biol 8: 931-937, 2007.

18 Kang R and Tang D: Autophagy in pancreatic cancer pathogenesis and treatment. Am J Cancer Res 2: 383-396, 2012.

19 Krysko DV, Garg AD, Kaczmarek A, Krysko O, Agostinis P and Vandenabeele P: Immunogenic cell death and DAMPs in cancer therapy. Nat Rev Cancer 12: 860-875, 2012.

20 Schnurr $M$ and Duewell P: Induction of immunogenic cell death by targeting RIG-I-like helicases in pancreatic cancer. Oncoimmunology 3: e955687, 2014.

21 Yang S, Wang X, Contino G, Liesa M, Sahin E, Ying H, Bause A, Li Y, Stommel JM, Dell'antonio G, Mautner J, Tonon G, Haigis M, Shirihai OS, Doglioni C, Bardeesy N and Kimmelman AC: Pancreatic cancers require autophagy for tumor growth. Genes Dev 25: 717-729, 2011.

22 Tang D, Kang R, Livesey KM, Cheh CW, Farkas A, Loughran P, Hoppe G, Bianchi ME, Tracey KJ, Zeh HJ, 3rd and Lotze MT: Endogenous HMGB1 regulates autophagy. J Cell Biol 190: 881892,2010

23 Tang D, Loze MT, Zeh HJ and Kang R: The redox protein HMGB1 regulates cell death and survival in cancer treatment. Autophagy 6: 1181-1183, 2010.

24 Kang R, Tang D, Loze MT and Zeh HJ: Apoptosis to autophagy switch triggered by the MHC class III-encoded receptor for advanced glycation endproducts (RAGE). Autophagy 7: 91-93, 2011.

25 Livesey KM, Kang R, Vernon P, Buchser W, Loughran P, Watkins SC, Zhang L, Manfredi JJ, Zeh HJ, 3rd, Li L, Lotze MT and Tang D: p53/HMGB1 complexes regulate autophagy and apoptosis. Cancer Res 72: 1996-2005, 2012.

26 Rosenfeldt MT, O'Prey J, Morton JP, Nixon C, MacKay G, Mrowinska A, Au A, Rai TS, Zheng L, Ridgway R, Adams PD, Anderson KI, Gottlieb E, Sansom OJ and Ryan KM: p53 status determines the role of autophagy in pancreatic tumour development. Nature 504: 296-300, 2013.

27 Kang R, Tang D, Schapiro NE, Livesey KM, Farkas A, Loughran P, Bierhaus A, Lotze MT and Zeh HJ: The receptor for advanced glycation end products (RAGE) sustains autophagy and limits apoptosis, promoting pancreatic tumor cell survival. Cell Death Differ 17: 666-676, 2010.

28 Mareninova OA, Hermann K, French SW, O'Konski MS, Pandol SJ, Webster P, Erickson AH, Katunuma N, Gorelick FS, Gukovsky
I and Gukovskaya AS: Impaired autophagic flux mediates acinar cell vacuole formation and trypsinogen activation in rodent models of acute pancreatitis. J Clin Invest 119: 3340-3355, 2009.

29 Aravindan S, Ramraj SK, Somasundaram ST and Aravindan N: Novel adjuvants from seaweed impede autophagy signaling in therapy-resistant residual pancreatic cancer. J Biomed Sci 22: 28, 2015.

30 Green DR, Ferguson T, Zitvogel L and Kroemer G: Immunogenic and tolerogenic cell death. Nat Rev Immunol 9: 353-363, 2009.

31 Kepp O, Senovilla L, Vitale I, Vacchelli E, Adjemian S, Agostinis P, Apetoh L, Aranda F, Barnaba V, Bloy N, Bracci L, Breckpot K, Brough D, Buque A, Castro MG, Cirone M, Colombo MI, Cremer I, Demaria S, Dini L, Eliopoulos AG, Faggioni A, Formenti SC, Fucikova J, Gabriele L, Gaipl US, Galon J, Garg A, Ghiringhelli F, Giese NA, Guo ZS, Hemminki A, Herrmann M, Hodge JW, Holdenrieder S, Honeychurch J, Hu HM, Huang X, Illidge TM, Kono K, Korbelik M, Krysko DV, Loi S, Lowenstein PR, Lugli E, Ma Y, Madeo F, Manfredi AA, Martins I, Mavilio D, Menger L, Merendino N, Michaud M, Mignot G, Mossman KL, Multhoff G, Oehler R, Palombo F, Panaretakis T, Pol J, Proietti E, Ricci JE, Riganti C, Rovere-Querini P, Rubartelli A, Sistigu A, Smyth MJ, Sonnemann J, Spisek R, Stagg J, Sukkurwala AQ, Tartour E, Thorburn A, Thorne SH, Vandenabeele P, Velotti F, Workenhe ST, Yang H, Zong WX, Zitvogel L, Kroemer G and Galluzzi L: Consensus guidelines for the detection of immunogenic cell death. Oncoimmunology 3: e955691, 2014.

32 Kroemer G, Galluzzi L, Kepp O and Zitvogel L: Immunogenic cell death in cancer therapy. Annu Rev Immunol 31: 51-72, 2013.

33 Stoetzer OJ, Wittwer C, Lehner J, Fahmueller YN, Kohles N, Fersching DM, Leszinski G, Roessner J and Holdenrieder S: Circulating nucleosomes and biomarkers of immunogenic cell death as predictive and prognostic markers in cancer patients undergoing cytotoxic therapy. Expert Opin Biol Ther 12(Suppl 1): S217-224, 2012.

34 Pilzweger $\mathrm{C}$ and Holdenrieder S: Circulating HMGB1 and RAGE as Clinical Biomarkers in Malignant and Autoimmune Diseases. Diagnostics (Basel) 5: 219-253, 2015.

35 Angelova AL, Grekova SP, Heller A, Kuhlmann O, Soyka E, Giese T, Aprahamian M, Bour G, Ruffer S, Cziepluch C, Daeffler L, Rommelaere J, Werner J, Raykov Z and Giese NA: Complementary induction of immunogenic cell death by oncolytic parvovirus H-1PV and gemcitabine in pancreatic cancer. J Virol 88: 5263-5276, 2014.

36 Mahadevan D and Von Hoff DD: Tumor-stroma interactions in pancreatic ductal adenocarcinoma. Mol Cancer Ther 6: 1186-1197, 2007.

37 Zhou H, Marks JW, Hittelman WN, Yagita H, Cheung LH, Rosenblum MG and Winkles JA: Development and characterization of a potent immunoconjugate targeting the Fn14 receptor on solid tumor cells. Mol Cancer Ther 10: 1276-1288, 2011.

38 Evans A, Lennard TW and Davies BR: High-mobility group protein 1(Y): metastasis-associated or metastasis-inducing? J Surg Oncol 88: 86-99, 2004.

39 Tarbe N, Evtimova V, Burtscher H, Jarsch M, Alves F and Weidle UH: Transcriptional profiling of cell lines derived from an orthotopic pancreatic tumor model reveals metastasis-associated genes. Anticancer Res 21: 3221-3228, 2001.

40 Nestl A, Von Stein OD, Zatloukal K, Thies WG, Herrlich P, Hofmann M and Sleeman JP: Gene expression patterns associated with the metastatic phenotype in rodent and human tumors. Cancer Res 61: 1569-1577, 2001. 
41 Huang QX, Wang GB, Sun NF and Wang CY: Inhibitory effects of high mobility group box 1 antisense nucleotide on invasion of human pancreatic cancer cell line PCNA-1. Ai Zheng 23: 10361040, 2004.

42 Takada M, Hirata K, Ajiki T, Suzuki Y and Kuroda Y: Expression of receptor for advanced glycation end products (RAGE) and MMP-9 in human pancreatic cancer cells. Hepatogastroenterology 51: 928-930, 2004.

43 Takada M, Koizumi T, Toyama H, Suzuki Y and Kuroda Y: Differential expression of RAGE in human pancreatic carcinoma cells. Hepatogastroenterology 48: 1577-1578, 2001.

44 Arumugam T, Ramachandran V, Gomez SB, Schmidt AM and Logsdon CD: S100P-derived RAGE antagonistic peptide reduces tumor growth and metastasis. Clin Cancer Res 18: 4356-4364, 2012.

45 Zhao T, Ren H, Jia L, Chen J, Xin W, Yan F, Li J, Wang X, Gao S, Qian D, Huang C and Hao J: Inhibition of HIF-1alpha by PX478 enhances the anti-tumor effect of gemcitabine by inducing immunogenic cell death in pancreatic ductal adenocarcinoma. Oncotarget 6: 2250-2262, 2015.

46 Kuramitsu Y, Wang Y, Kitagawa T, Tokuda K, Akada J, Tokunaga $\mathrm{M}$ and Nakamura K: High-mobility Group Box 1 and Mitogenactivated Protein Kinase activated Protein Kinase-2 Are Upregulated in Gemcitabine-resistant Pancreatic Cancer Cells. Anticancer Res 35: 3861-3865, 2015.

47 Kuramitsu Y, Wang Y, Taba K, Suenaga S, Ryozawa S, Kaino S, Sakaida I and Nakamura K: Heat-shock protein 27 plays the key role in gemcitabine-resistance of pancreatic cancer cells. Anticancer Res 32: 2295-2299, 2012.

48 Wittwer C, Boeck S, Heinemann V, Haas M, Stieber P, Nagel D and Holdenrieder S: Circulating nucleosomes and immunogenic cell death markers HMGB1, sRAGE and DNAse in patients with advanced pancreatic cancer undergoing chemotherapy. Int $\mathrm{J}$ Cancer 133: 2619-2630, 2013.

49 Boeck S, Wittwer C, Heinemann V, Haas M, Kern C, Stieber P, Nagel D and Holdenrieder S: Cytokeratin 19-fragments (CYFRA 21-1) as a novel serum biomarker for response and survival in patients with advanced pancreatic cancer. Br J Cancer 108: 1684 1694, 2013.
50 Yasuda T, Ueda T, Takeyama Y, Shinzeki M, Sawa H, Nakajima T, Ajiki T, Fujino Y, Suzuki Y and Kuroda Y: Significant increase of serum high-mobility group box chromosomal protein 1 levels in patients with severe acute pancreatitis. Pancreas 33: 359-363, 2006.

51 Bauden M, Pamart D, Ansari D, Herzog M, Eccleston M, Micallef J, Andersson B and Andersson R: Circulating nucleosomes as epigenetic biomarkers in pancreatic cancer. Clin Epigenetics 7: 106, 2015.

52 Takeda T, Izumi H, Kitada S, Uramoto H, Tasaki T, Zhi L, Guo X, Kawatsu Y, Kimura T, Horie S, Nabeshima A, Noguchi H, Wang KY, Sasaguri Y, Kohno K and Yamada S: The combination of a nuclear HMGB1-positive and HMGB2-negative expression is potentially associated with a shortened survival in patients with pancreatic ductal adenocarcinoma. Tumour Biol 35: 10555-10569, 2014.

53 Wittwer C, Boeck S, Heinemann V, Haas M, Stieber P, Nagel D and Holdenrieder S: Soluble receptor of advanced glycation end products (sRAGE) indicates response to chemotherapy in pancreatic cancer patients. Int J Clin Pharmacol Ther 51: 67-69, 2013.

54 Kang R, Zhang Q, Zeh HJ, 3rd, Lotze MT and Tang D: HMGB1 in cancer: good, bad, or both? Clin Cancer Res 19: 4046-4057, 2013.

55 El Gazzar M, Yoza BK, Chen X, Garcia BA, Young NL and McCall CE: Chromatin-specific remodeling by HMGB1 and linker histone $\mathrm{H} 1$ silences proinflammatory genes during endotoxin tolerance. Mol Cell Biol 29: 1959-1971, 2009.

56 Chung HW, Lim JB, Jang S, Lee KJ, Park KH and Song SY: Serum high mobility group box-1 is a powerful diagnostic and prognostic biomarker for pancreatic ductal adenocarcinoma. Cancer Sci 103: 1714-1721, 2012.
Received June 29, 2016

Revised July 17, 2016

Accepted July 18, 2016 